\title{
Simple Example of a Compact NonHausdorff Space with Closed Singletons
}

\author{
Yu-Lin Chou*
}

\begin{abstract}
We give as a main message a simple but "finer" example of a compact nonHausdorff space where every singleton is closed.
\end{abstract}

MSC 2020: 54B99; 54E99

Keywords: compactness; hypernonHausdorff spaces; singletons

Although every singleton, i.e. a set consisting of exactly one point, in a Hausdorff space is closed, not every space with closed singletons is Hausdorff.

We give in particular a simple example of a nonHausdorff space where every singleton is closed, with the space even being compact. What we are showing is more refined. A space $X$ with at least two points is called hypernonHausdorff if and only if $x, y \in X$ being distinct and $G_{1}, G_{2}$ being neighborhoods of $x$ and $y$ respectively imply $G_{1} \cap G_{2} \neq \varnothing$.

We prove

Theorem 1. There is some compact hypernonHausdorff space with all singletons being closed.

Proof. Let $X$ be an infinite set; let $\mathscr{T}_{X}:=\{\varnothing\} \cup\left\{G \mid G^{c}\right.$ is finite $\}$. Then $\mathscr{T}_{X}$ is a topology of $X$.

If $x, y \in X$ are distinct, and if $V_{1}, V_{2} \in \mathscr{T}_{X}$ are neighborhoods of $x$ and $y$ respectively with empty intersection, then $V_{1}^{c} \cup V_{2}^{c}=X$, which is a finite set by construction and also an infinite set by assumption; so $X$ is hypernonHausdorff.

Since $X \backslash\{x\} \in \mathscr{T}_{X}$ for all $x \in X$, every singleton in $X$ is closed.

To see that $X$ is compact, let $\left\{G_{\theta}\right\}_{\theta \in \Theta}$ be an open cover of $X$. Fix a finite subset $\Gamma$ of $\Theta$; then $X \backslash \bigcup_{\theta \in \Gamma} G_{\theta}$ is finite. If $x_{1}, \ldots, x_{n}$ are all the points of $X \backslash \bigcup_{\theta \in \Gamma} G_{\theta}$, choose $\theta_{1}, \ldots, \theta_{n} \in \Theta \backslash \Gamma$ such that $x_{i} \in G_{\theta_{i}}$ for all $1 \leq i \leq n$. But then we have obtained a finite subcover $\left\{G_{\theta}\right\}_{\theta \in \Gamma \cup\left\{\theta_{1}, \ldots, \theta_{n}\right\}}$ of $\left\{G_{\theta}\right\}_{\theta \in \Theta}$.

*Institute of Statistics, National Tsing Hua University, Hsinchu 30013, Taiwan, R.O.C.; Email: y.l.chou@gapp.nthu.edu.tw 
The proof of Theorem 1 immediately suggests

Proposition 1. There is some second-countable compact hypernonHausdorff space with all singletons being closed.

Proof. Let $X:=\mathbb{N}$, and topologize $X$ in the same way as in Theorem 1 . Then $X$ is by Theorem 1 a compact hypernonHausdorff space with all singletons being closed.

But $X$ is evidently second-countable; for instance, we may consider the countable collection $\{X \backslash\{1, \ldots, n\} \mid n \in \mathbb{N}\}$. 